\title{
Rigid structure of fractal aggregates of lysozyme
}

\author{
G. C. FADDA and D. LAIREZ \\ Laboratoire Léon Brillouin, CEA-CNRS CEA/Saclay \\ 91191 Gif-sur-Yvette cedex, France
}

(received 5 May 2000; accepted in final form 16 October 2000)

PACS. 87.50.Gi - Ionizing radiations (ultraviolet, X-rays, $\gamma$-rays, ions, electrons, positrons, neutrons, and mesons, etc.).

PACS. 61.43.Hv - Fractals; macroscopic aggregates (including diffusion-limited aggregates).

PACS. 81.10.-h - Methods of crystal growth; physics of crystal growth.

\begin{abstract}
The aggregation of hen egg-white lysozyme upon salt addition was studied by quasi-elastic light scattering. Our results agree with the fractal structure of the aggregates already reported in the literature. However, we also demonstrate that these aggregates are rigid, since they do not display any fluctuation of internal concentration. Such a rigid internal structure is a key point to reconcile the fractal structure of the aggregates and their colloid-like ordering. Furthermore, this result has to be considered for understanding crystal nucleation.
\end{abstract}

Introduction. - The determination of globular proteins structure by neutron or X-ray diffraction is an increasing activity in biophysics [1]. Crystallization of protein is a crucial point for these studies, although crystal growth methods remain greatly empirical up to now. The understanding of protein crystallization is thus of major importance and motivates the study of supersaturated protein solutions. A key point concerns the optimum conditions for nucleation to occur. It has been shown that under physicochemical conditions favorable to attractive interactions between lysozyme molecules and thus to nucleation, fractal aggregates appear in supersaturated solutions [2]. The point is to know whether fractal aggregation is a necessary initial stage for nucleation.

Recently, lysozyme fractal aggregates have been extensively studied [2,3]. Their structure and growth with time have been analyzed in terms of the diffusion-limited (DLCA) and reaction-limited (RLCA) cluster aggregation models. For instance, lysozyme at a concentration of $3 \mathrm{mM}$ at $20^{\circ} \mathrm{C}$ in a $p \mathrm{H} 4.5$ buffer containing $0.5 \mathrm{M} \mathrm{NaCl}$, forms fractal aggregates with a dimension $d_{\mathrm{f}}=2.08 \pm 0.14$. For increasing concentration, it has been shown [3] the existence of colloid-like ordering of these aggregates, as revealed by a maximum in the light scattering intensity as a function of scattering vector $q$. Such a result is quite puzzling. First, because the presence of salt prevents possible electrostatic repulsion. Secondly, because usually low-density fractals either interpenetrate or shrink as their concentration increases. Thus no interaction peak should be observed at low scattering vector.

In this paper, we report light scattering measurements on supersaturated lysozyme solutions. Experiments were carried out in physicochemical conditions quite similar to that of Georgalis et al. [2] and Umbach et al. [3]. We recover their results concerning the fractal structure of the aggregates, but our main observation is concerned with the dynamical structure factor which does not reveal any internal modes for the aggregates. This demonstrates that the aggregates have a rigid internal structure. Such a rigid structure explains their strong interactions.

(c) EDP Sciences 
Materials and methods. - Hen egg-white lysozyme was purchased from Sigma Chemicals. The experiments were conducted in a buffer containing $0.10 \mathrm{M}$ sodium acetate/acetic acid $(\mathrm{NaAc})$ at $p \mathrm{H}=4.5$. Lysozyme at the concentration of $60 \mathrm{mg} / \mathrm{ml}$ and $\mathrm{NaCl}$ at the concentration of $1.0 \mathrm{M}$ were dissolved separately in the buffer. Equal volumes of the two solutions were rapidly mixed and filtrated twice with Millipore GV $0.22 \mu \mathrm{m}$ filters. All measurements were carried out at $20^{\circ} \mathrm{C}$.

The experiments were performed with a home-made spectrometer using a vertically polarized argon gas laser with a wavelength $\lambda_{0}=488 \mathrm{~nm}$. The scattered intensity was measured in the horizontal plane at scattering angles $\theta$ from $20^{\circ}$ to $150^{\circ}$, corresponding to scattering vectors $q=\left(4 \pi n / \lambda_{0}\right) \times \sin (\theta / 2)$ between $5 \times 10^{-3}$ and $3.5 \times 10^{-2} \mathrm{~nm}^{-1}$. The total scattered intensity per volume unit of toluene was taken as reference. The scattering intensity of toluene was found to be independent of the scattering angle within 1\%. From the literature, the Rayleigh ratio of toluene is equal to $R_{\text {toluene }}=42.7 \times 10^{-6} \mathrm{~cm}^{-1}$. The absolute value (in $\mathrm{cm}^{-1}$ ) of the scattering cross-section per volume unit, $\widetilde{\Sigma}(q)$, of lysozyme in solution was deduced from the ratio $\widetilde{\Sigma}(q)=\left(\left(I(q)-I_{0}\right) / I_{\text {toluene }}\right) \times R_{\text {toluene }}$, where $I(q), I_{0}, I_{\text {toluene }}$ are the scattered intensities of the lysozyme solution, the pure solvent and toluene, respectively. The refractive index increment of lysozyme in water is equal to $\mathrm{d} n / \mathrm{d} C=0.230 \mathrm{~cm}^{3} \mathrm{~g}^{-1}[4]$, where $C\left(\mathrm{in} \mathrm{g} / \mathrm{cm}^{3}\right)$ is the lysozyme concentration. The light scattering contrast factor $K^{2}=\left(2 \pi n_{0} / \lambda_{0}^{2} N_{\mathrm{a}} \times \mathrm{d} n / \mathrm{d} C\right)^{2}$, where $N_{\mathrm{a}}$ is Avogadro's number and $n_{0}=1.3330$ the refractive index of water, was calculated to be $1.09 \times 10^{-6} \mathrm{~cm}^{2} \mathrm{~g}^{-2} \mathrm{~mol}$. This allows us to calculate the quantity $\widetilde{\Sigma}(q) /\left(K^{2} \times C\right)$ (in $\mathrm{g} / \mathrm{mole}$ ) and to compare it to the molecular weight, $M=14300 \mathrm{~g} / \mathrm{mole}$, of lysozyme molecules. Quasi-elastic light scattering (QELS) measurements were performed in the selfbeating mode. The dynamical structure factor $S(q, t)$ was deduced from the autocorrelation function of the time-dependent scattered intensity $I(t)$ computed by a 256 channels Malvern 7032 multi-correlator.

Quasi-elastic light scattering and internal modes. - The experimental results discussed in the following are concerned with QELS measurements. Let us do a summary of what it is theoretically expected for dilute solutions of large particles in a $q$-range for which only one characteristic length (the size of the particle) is relevant.

For solutions, $S(q, t)$ represents the relaxation over the length $q^{-1}$ of concentration fluctuations. In dilute solution and at large $q^{-1}$ compared to the characteristic size $R_{\mathrm{g}}$ of the particles $\left(q R_{\mathrm{g}}<1\right)$, this relaxation is achieved via the self-diffusion of the particles by translational motion. In the case of monodisperse particles with a diffusion coefficient $D, S(q, t)$ is a simple exponential decay with a characteristic time $\tau$ corresponding to the time $1 /\left(D q^{2}\right)$ needed for molecules to cover the $q^{-2}$ area: $S(q, t)=e^{-D q^{2} t}$. For dense particles and for fractals made of hydrodynamically interacting units (Zimm model), the particle encounters a friction $f$ proportional to its radius, $f=6 \pi \eta R_{\mathrm{H}}$, where $R_{\mathrm{H}}=\alpha R_{\mathrm{g}}$ is the hydrodynamic radius and $\eta$ the solvent viscosity. The proportionality factor $\alpha$ depends on particle conformation but it is never far from unity [5].

For increasing scattering vector $q\left(q R_{\mathrm{g}}>1\right)$, different cases have to be distinguished. For dense particles such as colloids and spherical micelles, or for particles having a rigid structure, internal density and concentration fluctuations are negligible and $S(q, t)$ is dominated by the translational motion. For fractals having a flexible structure, translational motion of the particle still contributes to $S(q, t)$, but in practice $S(q, t)$ is dominated by concentration fluctuations inside the fractal itself. Let us use a scaling argument stating that in this $q$ range $\left(q R_{\mathrm{g}}>1\right), S(q, t)$ is insensitive to the overall size of the fractal. It comes necessarily that the diffusion coefficient corresponds to the motion of fractal segments having a size $q^{-1}$. In the 
case of hydrodynamic interactions between fractal segments (Zimm model) one can write

$$
D\left(q R_{\mathrm{g}}\right)=\frac{k T}{6 \pi \eta R_{\mathrm{H}}} F\left(q R_{\mathrm{g}}\right) \text { with }\left\{\begin{array}{l}
F\left(q R_{\mathrm{g}}<1\right)=1 \\
F\left(q R_{\mathrm{g}}>1\right)=q R .
\end{array}\right.
$$

Actually, for $q R_{\mathrm{g}}>1$, all the fractal segments having a size smaller than $q^{-1}$ participate to the dynamical structure factor. Due to the distribution in size of these segments, $S(q, t)$ is no longer exponential. As time goes by, the segments shorter than $(t \times k T / \eta)^{1 / 3}$ have already relaxed, but the slower ones have not. This distribution in relaxation time is responsible for a stretched exponential behavior of $S(q, t)[6]$ :

$$
S\left(q R_{\mathrm{g}}>1, t\right)=e^{-\left(D(q) q^{2} t\right)^{2 / 3}}
$$

These two equations are the signature of internal modes and have been checked by experiments on various objects: binary mixture near the critical point (except for the nonexponential relaxation) [7], linear polymers in good and $\theta$ solvent [8-11], branched polymers [12,13], associative polymers aggregates [14], dilute solution of bilayers [15]. This is the general behavior of objects having a flexible structure allowing fluctuations of internal concentration. Note that in the case of no hydrodynamic interactions between fractal segments (Rouse model), an even more drastic effect is expected for internal modes: $D(q) \propto q^{2}$ and $S\left(q R_{\mathrm{g}}>1, t\right)=e^{-\left(D(q) q^{2} t\right)^{1 / 2}}$.

Results. - After salt addition, lysozyme aggregates. The aggregation kinetics has been extensively studied $[2,3]$ and is not the purpose of this paper. The aggregation process is very slow in the physicochemical conditions here studied. In fig. 1, the reduced scattering cross-section $\widetilde{\Sigma}(q) /\left(K^{2} \times C \times M\right)$ is plotted as a function of the scattering vector $q$, for a solution at the initial stage and 4 days later. Initially, the spectrum is completely flat because of the very small radius of gyration of lysozyme compared to the light scattering $q$ range. The mean value is $\left\langle\widetilde{\Sigma}(q) /\left(K^{2} \times C \times M\right)\right\rangle=1.35 \pm 0.05$. To explain this value higher than unity, one needs to take into account attractive lysozyme-lysozyme interactions. In the dilute regime, the osmotic compressibility can be expressed as a virial expansion leading to $\widetilde{\Sigma}(0) /\left(K^{2} \times C \times M\right)=$ $1-2 M A_{2} C+\ldots$, where $A_{2}$ is the second virial coefficient. Negative values for $A_{2}$ have already been observed. With $0.5 \mathrm{M} \mathrm{NaCl}$ in water the value $M A_{2}=-6.4 \mathrm{~cm}^{3} \mathrm{~g}^{-1}$, given in ref. [4, 16], leads to the expected value $\widetilde{\Sigma}(0) /\left(K^{2} \times C \times M\right)=1-2 M A_{2} C=1.38$, in good agreement with our result.

Four days later, the scattering cross-section has increased strongly at low $q$ values (see fig. 1). This reveals association of lysozyme molecules in large aggregates. However, at high $q$ values the scattering spectrum superimposes on the one measured at the beginning. This means that only a very small amount of lysozyme molecules are involved in the aggregates, and the contribution of the free lysozyme molecules to the total scattered intensity cannot be neglected. This is the reason why the scattering function of the aggregates cannot be directly deduced from these measurements.

QELS measurements were performed on lysozyme solutions after aggregation has occurred. In fig. $2, S(q, t)$ measured at different scattering vectors $q$ is plotted as a function of $t q^{2}$. Such a plot would give a single master curve in the case of a single diffusion process with a diffusion coefficient $D: S(q, t)=e^{-D t q^{2}}$. In the present case, relaxation of concentration fluctuations shows two modes with characteristic times differing by two orders of magnitude. The different curves superimpose at short and large $t q^{2}$ values. Without further data analysis, this indicates that the two relaxation modes are controlled by diffusion. In order to account more quantitatively for these two modes, $S(q, t)$ is written as the sum of two exponential decays:

$$
S(q, t)=Y_{1}(q) e^{-D_{1} t q^{2}}+Y_{2}(q) e^{-D_{2} t q^{2}},
$$




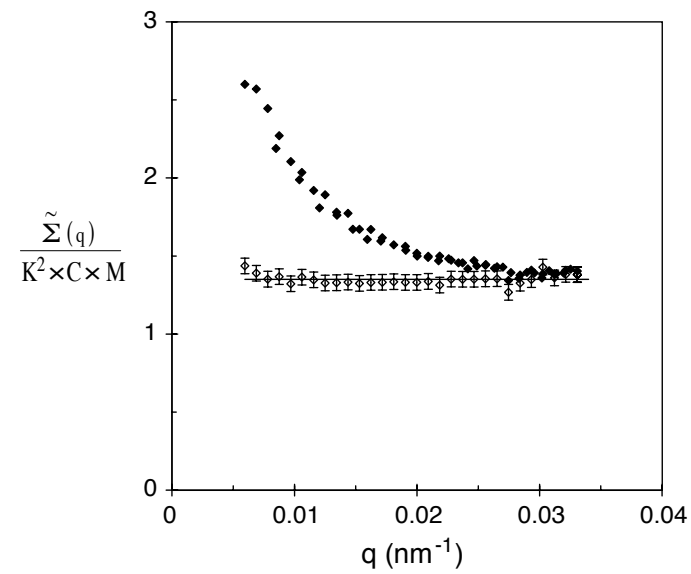

Fig. 1

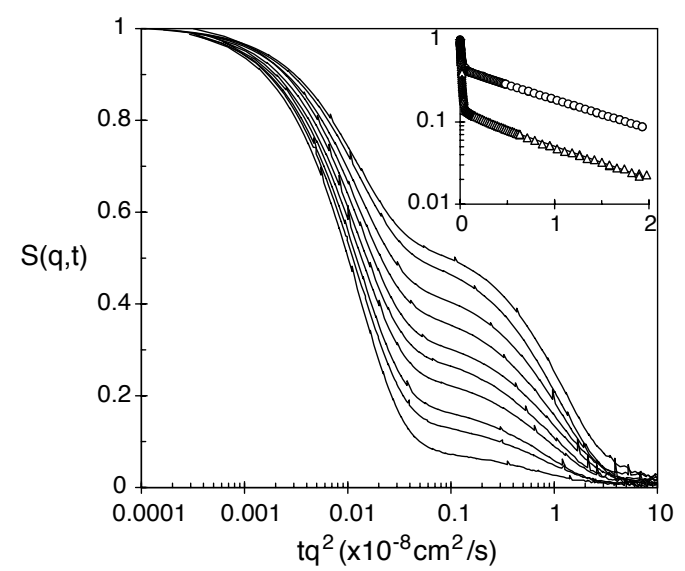

Fig. 2

Fig. 1 - Reduced scattering cross-section per volume unit $\widetilde{\Sigma}(q) /\left(K^{2} \times C \times M\right)$ vs. scattering vector $q$ measured for lysozyme concentration $C=3 \times 10^{-2} \mathrm{~g} / \mathrm{cm}^{3},[\mathrm{NaCl}]=0.5 \mathrm{M}, T=20^{\circ} \mathrm{C}$ and $p \mathrm{H}=4.5$. Hollow symbols: measurement at the initial stage, full symbols: 4 days later. The straight line corresponds to the mean initial value $1.35 \pm 0.05$.

Fig. 2 - Dynamical structure factor $S(q, t)$ vs. $t q^{2}$ in a linear-log scale (inset: log-linear scale) for different scattering vectors $q$. From upper to lower curve $q=5.9,7.4,8.9,10,12,13,14,17,20$ and $26 \times 10^{-3} \mathrm{~nm}^{-1}$. In the inset $\left(q=8.9,20 \times 10^{-3} \mathrm{~nm}^{-1}\right.$ upper and lower curve, respectively) the loglinear scale outlines more particularly the long-time part of the relaxation which decays exponentially.

where the subscripts 1 and 2 refer to short- and long-time relaxation modes, respectively.

The short-time diffusion coefficient $D_{1}$ is found to be $q$-independent and equal to $(76 \pm$ $1.5) \times 10^{-8} \mathrm{~cm}^{2} / \mathrm{s}$. Using the Stockes-Einstein relation $D_{1}=k T /\left(6 \pi \eta R_{\mathrm{H} 1}\right)$, the hydrodynamic radius $R_{\mathrm{H} 1}=(2.03 \pm 0.05) \mathrm{nm}$ reported in the literature [17] would lead to $D_{1}=106 \times$ $10^{-8} \mathrm{~cm}^{2} / \mathrm{s}$ for free lysozyme molecules. Here again the discrepancy between the measured and the calculated values is explained by lysozyme-lysozyme interactions. For dilute solutions, the diffusion coefficient can be written as $D=D_{0}\left(1+k_{D} C\right)$, where $k_{D}$ is an effective twobody interaction parameter reflecting both thermodynamic and hydrodynamic interactions. Usually in good solvent, $k_{D}$ is very small because of cancellation of thermodynamic and hydrodynamic interactions, but here the second virial coefficient is negative and thus magnifies the concentration effects. It has been reported [4] that $k_{D}=-10 \mathrm{~cm}^{3} \mathrm{~g}^{-1}$ for lysozyme solutions in conditions identical to those of this study. This value gives $D=74 \times 10^{-8} \mathrm{~cm}^{2} / \mathrm{s}$ at $C=30 \mathrm{mg} / \mathrm{cm}^{3}$, in good agreement with our $D_{1}$ value.

The long-time diffusion coefficient is found to be $D_{2}=(0.87 \pm 0.07) \times 10^{-8} \mathrm{~cm}^{2} / \mathrm{s}$. It can be ascribed to the presence of lysozyme aggregates. The corresponding hydrodynamic radius is $R_{\mathrm{H} 2}=(245 \pm 20) \mathrm{nm}$. Such a high value requires two remarks.

1) As shown in fig. $3, D_{2}$ is $q$-independent within the experimental accuracy. This means that the translational diffusion motion of aggregates is probed even at length scales smaller than their size. In other words, there are no concentration fluctuations inside the aggregates (no internal modes). In fig. 3, the measurements are compared to the behavior expected for internal modes.

2) At large $t q^{2}$ values, $S(q, t)$ is nicely described by a single exponential and not by a stretched exponential as expected in the case of internal modes for flexible structures. This feature is emphasized in the inset of fig. 2, where $S(q, t)$ is plotted as a function of $t q^{2}$ in a log-linear scale. 


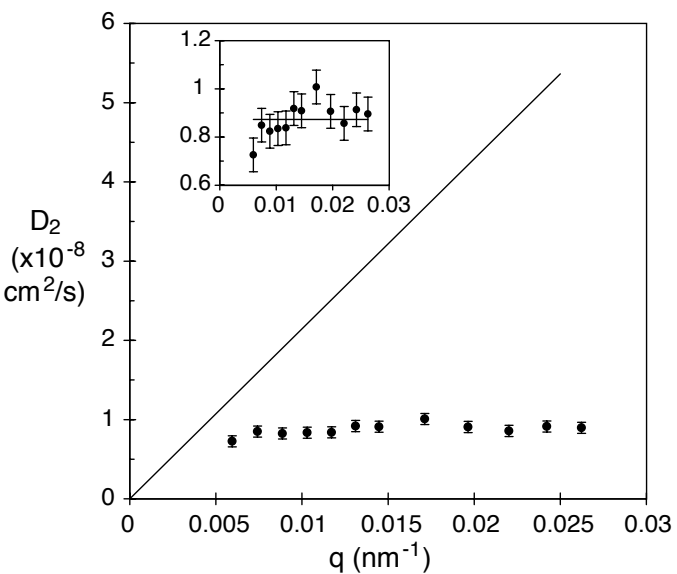

Fig. 3

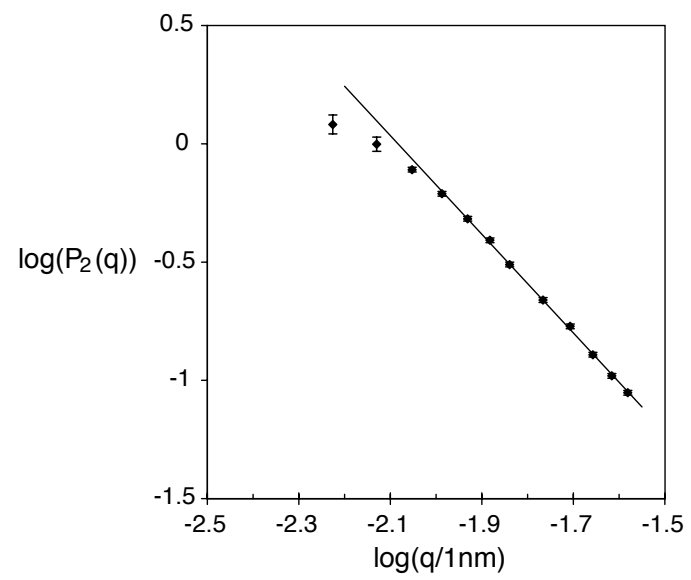

Fig. 4

Fig. 3 - Diffusion coefficient $D_{2}(q)$ of the aggregates as a function of the scattering vector $q$. The straight line has a slope equal to $k T / 6 \pi \eta$, with $\eta$ the solvent viscosity, and corresponds to what would be expected for relaxation of internal concentration fluctuations.

Fig. 4 - Form factor $P_{2}(q) \propto Y_{2}(q) / Y_{1}(q)$ of lysozyme aggregates deduced from the $q$-dependent amplitudes of the two relaxation modes observed by QELS. The straight line has a slope equal to -2 .

The two above observations prove that there is no fluctuation of internal concentration inside the aggregates. There are only two possible explanations: 1) the aggregates have a dense structure, i.e. they are not fractal; 2) the aggregates are fractal but rigid. Of course, previous experiments reported in the literature plead in favor of this second possibility, but it has to be checked in our case to rule out artifact. This can be achieved by analyzing the amplitudes of the two relaxation modes [14]. In a dilute regime, cross terms can be neglected in the total scattered intensity, $I(q)$, so that

$$
I(q)=I_{1}(q)+I_{2}(q)
$$

where $I_{1}(q)$ and $I_{2}(q)$ are the scattering intensities corresponding to free lysozyme and aggregates, respectively. In eq. (3), each mode is weighted by the ratio of the contribution of a given species to the total scattered intensity:

$$
Y_{1}(q)=\frac{I_{1}(q)}{I(q)}, \quad Y_{2}(q)=\frac{I_{2}(q)}{I(q)} .
$$

Due to their small size, the contribution $I_{1}$ of free lysozymes is $q$-independent (see fig. 1 , hollow symbols). Neglecting interactions between aggregates, the scattering contribution $I_{2}(q)$ is proportional to their form factor $P_{2}(q)$ which can thus be deduced from the ratio

$$
P_{2}(q) \propto I_{2}(q) \propto \frac{Y_{2}(q)}{Y_{1}(q)} .
$$

The result is plotted in fig. 4 in log-log scales. We found that $P_{2}(q) \propto q^{-2}$ at $q^{-1}$ smaller than the size of the aggregates. This result is in good agreement with the fractal dimension, $d_{\mathrm{f}}$, of lysozyme aggregates already reported [2]: $1.97<d_{\mathrm{f}}<2.05$ depending on lysozyme concentration with $0.5 \mathrm{M} \mathrm{NaCl}$. Note that in the case of a dense structure, one would expect rather a Porods law $\left(S_{2}(q) \propto q^{-4}\right)$ as the signature of a sharp interface between the inside and the outside of the aggregates [18].

Discussion and conclusion. - Our QELS data have been interpreted in terms of largeaggregates self-diffusion rather than of a slow cooperative process. Fractal aggregates being 
so large, one may wonder about their possible interactions. Let us show a posteriori that our analysis is self-consistent and that these interactions can be neglected. The aggregates are found to have a radius $10^{2}$ times higher than the one of lysozyme. One can estimate the number of lysozyme molecules per aggregate to be $N=\left(R_{\mathrm{H} 2} / R_{\mathrm{H} 1}\right)^{d_{\mathrm{f}}}=10^{4}$. Our static and QELS measurements are sensitive to both the aggregates and the free molecules (see fig. 1 and fig. 2), this means that the products of the molecular mass by the mass concentration for the aggregates and for free lysozyme molecules are of the same order. Consequently, the aggregates are $10^{4}$ times heavier than lysozyme molecules, but their concentration is $10^{4}$ times lower: $C(N)=C(1) \times 10^{-4}$. Let us define the overlap concentration $C^{*}$ of the aggregates as the ratio of their mass to the third power of their radius. $C^{*}$ scales as $C^{*}(N)=C^{*}(1) \times N^{1-3 / d_{\mathrm{f}}}=$ $C^{*}(1) \times N^{-1 / 2}$, where $C^{*}(1)$ is the overlap concentration of lysozyme. Finally, the volume fraction occupied by the aggregates is $C(N) / C^{*}(N)=\left[C(1) / C^{*}(1)\right] \times 10^{-2}$, which means that the aggregates are in a much more dilute regime than free lysozyme molecules. This supports our interpretation neglecting aggregate-aggregate interactions.

The lysozyme aggregates studied here are very large compared to the light scattering $q$ range. Thus, their internal structure and dynamics is probed. Actually, the static structure factor is found to vary as $q^{-2}$ as expected for fractals at $q R_{\mathrm{g}}>1$. In the same $q$ range, we find that the dynamic structure factor is not governed by internal modes. This observation proves that lysozyme fractal aggregates are rigid. This result explains the colloid-like ordering of these aggregates already reported in the literature [3] and revealed by a scattered intensity peak at low $q$. Apart from this rigidity, such a peak could be caused by electrostatic interaction (as for polyelectrolytes [19]) or "hard sphere"-like interactions (as for star polymers [20,21] or spherical polymer micelles [22] which both display liquid-like ordering). The former is to be ruled out because of the very high salt concentration but the latter needs special attention. Because of their low-density fractal structure, polymer coils are able to interpenetrate. Thus, polymers in good solvent $\left(d_{\mathrm{f}}=1.7\right)$ or in $\theta$ solvent $\left(d_{\mathrm{f}}=2\right)$ pass from a dilute regime (with a distance $d$ between coils larger than their size $R$ ) to a semi-dilute regime (with $d<R$ ) with an overall scattered intensity profile remaining unchanged but with a decreasing characteristic size $\xi(\xi \propto R$ in the dilute regime, and $\xi \propto d$ in a semi-dilute one). However, the structure of lysozyme aggregates is different from that of a linear chain and rather similar to a branched structure. Such a structure is comparable with monodisperse randomly branched polymers in good solvent which have the same fractal dimension $d_{\mathrm{f}}=2$ and have been well studied from the point of view of the concentration behavior. Theoretically [23], monodisperse branched polymers do not interpenetrate. The reason is that if they interpenetrate, excluded-volume interaction would be screened and thus the fractal dimension would be that of a Cayley tree [24] $\left(d_{\mathrm{f}}=4\right)$ which is not possible in three dimensions. Actually, in the reaction bath, within which they are growing, branched polymers of a given size are swollen by smaller ones, leading to $d_{\mathrm{f}}=2.5$. Once monodisperse, this possibility is removed and branched polymers do not interpenetrate but rather shrink for increasing concentration. This shrinkage is enabled by the soft and flexible structure of the polymer. Experimentally [25], despite a different mechanism, the concentration behavior of monodisperse branched polymers is similar to that of linear polymers (except for a different concentration dependence of the correlation length). Lysozyme aggregates behave in a completely different manner and present liquid-like interactions. This can be fully explained taking into account their rigidity.

From our quasi-elastic data, we find that the aggregates are monodispersed (as shown by the simple exponential decay of $S(q, t)$ at long time, see fig. 2). On the other hand, previous results have shown that their fractal dimension is $2.08 \pm 0.14$ [2]. These two results come into conflict with respect to DLCA or RLCA models. The former predicts monodisperse aggregates but with a fractal dimension lower than 2. The latter predicts a fractal dimension in better 
agreement with the one of lysozyme aggregates but with a polydispersity which should have an incidence on $S(q, t)[26]$. In summary, lysozyme aggregates are monodisperse fractals with a dimension of the order of 2 and a rigid structure.

The reason for this rigidity is not understood and should be investigated. In our opinion it is a key point for the understanding of lysozyme crystal nucleation. If fractal aggregation is a necessary initial stage for nucleation, a rearrangement of lysozymes inside the aggregates is required for a nucleus to appear. Clearly, the aggregate rigidity which causes the lack of fluctuations is not favorable to such a rearrangement.

We warmly thank O. VIDAL for having kindly communicated to us her knowledge about lysozyme and P. CALMETTES for interesting remarks about the paper.

\section{REFERENCES}

[1] See for instance Crystallization of Nucleic Acids and Proteins, edited by A. Ducruix and R. GIEGÉ, 2nd edition (Oxford University Press, New York) 1999.

[2] Umbach P., Georgalis Y. and Saenger W., J. Am. Chem. Soc., 120 (1998) 2382.

[3] Georgalis Y., Umbach P., Saenger W., Ihmels B. and Soumpasis D. M., J. Am. Chem. Soc., 121 (1999) 1627.

[4] Mushol M. and Rosenberger F., J. Chem. Phys., 103 (1995) 10424.

[5] Oono Y. and Конмото M., J. Chem. Phys., 78 (1983) 520.

[6] Dubois-Violette E. and de Gennes P.-G., Physics, 3 (1967) 181.

[7] Berge P., Calmettes P., Laj C. and Volochine B., Phys. Rev. Lett., 23 (1969) 302.

[8] Adam M. and Delsanti M., J. Phys. (Paris) Lett., 38 (1977) L-271.

[9] Martin J. E., Wilcoxon J. P. and OdineK J., Macromolecules, 25 (1992) 4635.

[10] Adam M., Lairez D., Raspaud E. and Farago B., Phys. Rev. Lett., 77 (1996) 3673.

[11] Adam M., Farago B., Schleger P., Raspaud E. and Lairez D., Macromolecules, 31 (1998) 9213.

[12] Martin J. E. and Wilcoxon J. P., Phys. Rev. Lett., 61 (1988) 373.

[13] Adam M., Lairez D., Karpasas M. and Gottlieb M., Macromolecules, 30 (1997) 5920.

[14] Raspaud E., Lairez D., Adam M. and Carton J.-P., Macromolecules, 27 (1994) 2956.

[15] Kimura Y., Oizumi J. and Hayakawa R., Mol. Cryst. Liq. Cryst. Sci, 332 (1999) 3069.

[16] Gripon C., Legrand L., Rosenman I., Vidal O., Robert M. C. and Boué F., J. Cryst. Growth, 178 (1997) 575.

[17] Svergun D. I., Richard S., Koch M. H. J., Sayers Z., Kuprin S. and Zaccai G., Proc. Natl. Acad. Sci. USA, Biophysics, 95 (1998) 2267 (in this paper the authors report for the radius of gyration $R_{\mathrm{g}}=(1.54 \pm 0.02) \mathrm{nm}$. We deduced $R_{\mathrm{H}}$ from the product $\left.R_{\mathrm{H}}=(5 / 3)^{1 / 2} \times R_{\mathrm{g}}\right)$; Eberstein W., Georgalis Y. and Saenger W., J. Cryst. Growth, 143 (1994) 71 (in this paper the value $R_{\mathrm{H}}=(2.09 \pm 0.08) \mathrm{nm}$ is reported $)$.

[18] Auvray L. and Auroy P., Neutron, X-ray and Light Scattering, edited by P. Lindner and Th. Zemb (North-Holland) 1991.

[19] Morfin I, Reed W. F., Rinaudo M. and Borsali R., J. Phys. II, 4 (1994) 1001.

[20] Adam M. and Lairez D., Fractals, 1 (1993) 149.

[21] Willner L., Jucknischke O., Richter D., Farago B., Fetters L. J. and Huang J. S., Europhys. Lett., 4 (1992) 297.

[22] Adam M., Carton J.-P., Corona-Vallet S. and Lairez D., J. Phys. II, 6 (1996) 1781.

[23] Daoud M. and Joanny J.-F., J. Phys., 42 (1981) 1359.

[24] Flory, P. J., in Principles of Polymer Chemistry (Cornell University Press, Ithaca) 1953.

[25] Delsanti M., Munch J.-P., Durand D., Busnel J.-P. and Adam M., Europhys. Lett., 13 (1990) 697.

[26] Martin J. E., Phys. Rev. A, 36 (1987) 3415. 